\title{
A Review of Remdesivir Use in End Stage Renal Disease (ESRD)
}

\section{Okelue Edwards Okobi1 ${ }^{*}$, Jennifer Dorce Medard1, Seneca Harberger1, Giselle Falconi ${ }^{1}$, Tobechukwu Joseph 0 kobi $^{2}$, Isioma Francis Okobi ${ }^{4}$, Rita Kimble Okobi ${ }^{3}$, Heden Presendieu, ${ }^{1}$, Manoj Bhattarai', Thomas Elimihele ${ }^{2}$, Bryan Dawkins ${ }^{1}$, Ishan Gunawardene ${ }^{1}$}

\author{
${ }^{1}$ Lakeside Medical Center, Belle Glade, Florida USA \\ 2University of District of Columbia, DC, USA \\ 3University of Maryland Global campus, College Park, USA \\ ${ }^{4}$ Northeastern University Boston Massachusetts \\ *Corresponding author details: Okelue Edwards. Okobi, MD; \\ drokelue.e.okobi@gmail.com
}

\begin{abstract}
The novel coronavirus disease 2019 (COVID-19) spread quickly worldwide following the first report of pneumonia of unknown cause in Wuhan, China. Since then, the incidence and mortality have continued to rise, with over 120 million cases of COVID-19 globally. The agency for Food and Drug Administration approved Remdesivir for the treatment of COVID-19, in October 2020. This approval supports the use of Remdesivir to treat SARS-CoV-2 infection in adults and children $\geq 12$ years of age who weigh $\geq 40 \mathrm{~kg}$. The use of Remdesivir in the treatment of COVID-19 patients was timely considering the rapid rate of spread and mortality from the disease, especially among elderly patients and those with existing comorbidities, including renal disease. There have been reports of increased mortality rate in patients with acute kidney injury (AKI) requiring renal replacement therapy following exposure to the virus. While Remdesivir has been considered safe and effective in the treatment of SARS-CoV-2 infection, recommendations have been issued against its use in End-Stage Renal Disease (ESRD) - patients with glomerular infiltration rate (eGFR) $<30 \mathrm{~mL} / \mathrm{min} / 1.73 \mathrm{~m}^{2}$. This is due to minimal safety data from clinical trials involving the use of Remdesivir in patients with ESRD and concerns of liver necrosis and renal tubule obstruction from earlier animal studies. This review focuses on existing literature on the use of patients with ESRD and their outcomes. While further studies are needed to prove the safety of Remdesivir among patients with ESRD, available literature shows that the advantages of short-duration therapy (5-10 days) outweigh the risk, especially when combined with hemodialysis and constant monitoring of Remdesivir concentration.
\end{abstract}

Keywords: Remdesivir; end-stage renal disease (ESRD); covid-19; acute kidney injury (AKI)

\section{INTRODUCTION}

The novel coronavirus disease 2019 (COVID-19) spread quickly around the world following the first report of pneumonia of unknown cause in Wuhan, China. Currently, there are over 98 million cases of COVID-19 globally (World Health Organization, 2021), with the rates still rising. The new disease has attracted scientists' attention because of its high mortality rate among older adults and individuals suffering from chronic diseases, including chronic kidney disease (CKD). Although numerous studies have recommended approaches for managing COVID-19 in patients with CKD, these patients' impact is still a challenge worldwide.

In October 2020, the FDA approved Remdesivir to treat SARS-CoV-2 infection (FDA, 2020). In late 2020 (Beigel, 2020), the final report on Remdesivir use in COVID-19 was released; the ACTT-1 clinical trial evaluated 1062 patients and showed that Remdesivir was a superior placebo in shortening the time to recovery in adults hospitalized with COVID-19.
This approval authorized the use of Remdesivir to treat SARS-CoV- 2 infection in adults and children $\geq 12$ years of age who weigh $\geq 40 \mathrm{~kg}$ but not in patients with glomerular filtration rate (eGFR) $<30 \mathrm{~mL} / \mathrm{min} / 1.73 \mathrm{~m}^{2}$ (Adamsick, 2020) due to minimal safety data in these patients.

Replication of the single-stranded RNA genome of SARSCoV-2 depends on RNA-dependent RNA polymerase (RDRP). Remdesivir is a prodrug that, after being metabolized, acts as an analog of ATP competing for incorporation by RDRP and thus interfering with viral RNA replication (Sheahan, 2017). The initial use of Remdesivir was as an investigational agent for Ebola, but applications for MARS and SARS have been reported as well. Intracellular Remdesivir prodrug (GS-5734) is converted into its alanine metabolite GS-704277 and subsequently into monophosphate and after its dephosphorylation yields the Remdesivir nucleoside core GS-441524. This is the predominant circulating metabolite of the drug. Studies have measured both levels of Remdesivir and GS-441524 to understand the excretion and circulation of the drug (FDA, 2020). 
Remdesivir has limited water solubility; therefore, the intravenous preparation contains a lyophilized excipient called sulfobutylether beta-cyclodextrin (SBECD). SBECD is a large oligosaccharide excreted through glomerular filtration (Kiser, 2020) with a $t_{1 / 2}$ elimination of $<2$ hours in individuals with normal kidney function. The concern with SBECD is that animal studies showed liver necrosis and renal tubule obstruction at doses $>250 \mathrm{mg} / \mathrm{kg}$ per day. Each $100 \mathrm{mg}$ of lyophilized powder and solution of Remdesivir contain 3-6g of SBECD, well below the maximum recommended dosage. Renal clearance of SBECD decreases in patients with ESRD and raises a concern in this category of patients. (Hoover et al., 2018) In a randomized controlled trial, Kiser et al. demonstrated renal clearance of SBECD after continuous venovenous hemofiltration. When used in patients with CKD, the accumulation of SBECD only occurs when dialysis was held for prolonged periods (Hafner, 2010).

Treatment with Remdesivir shows significant benefits in patients with COVID-19, mostly in severe cases (Gérard, 2020). In ESRD., guidelines are necessary to determine the safety of Remdesivir.

\section{METHODOLOGY}

Since the FDA approved the use of Remdesivir in COVID19 , questions remain about its safety in patients with ESRD. The objective is to report the use of Remdesivir in patients with ESRD and COVID-19, their outcomes, and toxicities associated. A systematic review of relevant literature was done using PubMed, Google Scholar, and Cochrane Library. The inclusive criteria were articles published on the correlation of ESRD patients on treatment for COVID-19 with Remdesivir. The keywords for our search were "ESRD," "dialysis," "Remdesivir," "CKD," "COVID-19," and all possible combinations of them A total of 10 results were matched to our keywords; then, articles focusing specifically on ESRD and Remdesivir were selected; resulting in 6 articles chosen for this review. Each article was selected using a two-step process; screening titles and abstract first, then assessing full text of records after. Key findings were extracted corresponding to the central questions of our review. The discussion of the results focused on what the suggested practices and common findings globally are.

\section{Results and discussion}

In a prospective observational study in 2020, Aiswarya et al. evaluated 48 patients with chronic kidney disease (CKD) requiring hemodialysis who tested positive for SARS-CoV-2 infection with moderate to severe presentation and who received one or more doses of Remdesivir. The treatment with Remdesivir was given at a dose of $2.5 \mathrm{mg} / \mathrm{kg}$ of edema-free body weight to a maximum dose of $100 \mathrm{mg}$ four hours before a hemodialysis session. A maximum of 6 doses was given in total for each patient. Liver function tests were monitored daily, and further Remdesivir doses were withheld if the levels of alanine aminotransferase (ALT) were $>5$ times higher than the normal upper limit or if the elevation was accompanied by signs or symptoms of liver inflammation. Nothing in this publication was reported on renal parameters. Besides Remdesivir, patients also received low molecular weight heparin (LMWH) subcutaneous and dexamethasone for 5 days. Nasopharyngeal swabs for COVID-19 by reverse transcription-polymerase reaction were repeated every 72 hours. After the administration of Remdesivir, there was a significant decline in CRP levels across all patients, independent of the disease's severity. Patients that received Remdesivir within the first 48 hours decreased the mean duration of hospitalization by 5.5 days compared to those who received it after. This study concluded that Remdesivir use in ESRD conferred clinical benefit in shorter recovery time and discharge time (Aiswarya et al. 2020).

Sorgel et al. in 2020 conducted a report on the pharmacokinetics of Remdesivir and its metabolites and treatment outcomes in patients with ESRD). Utilizing the premise that renal excretion of Remdesivir in healthy individuals is about $10 \%$ unchanged drug and $50 \%$ as the Remdesivir nucleoside core GS-441524 (FDA, 2020). Sorgel and his team followed a 70-year-old male patient with ESRD admitted to the hospital for COVID-19. Initially, the patient presented with typical pulmonary changes, but the clinical course deteriorated quickly and was transferred to ICU. Remdesivir was not considered at the onset since it is not recommended in patients with an estimated glomerular infiltration rate $<30 \mathrm{~mL} / \mathrm{min}$. Following transfer to the ICU, the patient continued deteriorating; thus, the medical team decided to commence treatment with Remdesivir. During the treatment, concentrations of Remdesivir and GS-441524 were measured. Concentrations of Remdesivir decreased rapidly after the first infusion. GS441524 concentrations decreased and then remained constant. Exposure for Remdesivir and GD-441524 was about 3-fold and 6-fold higher in the patient compared to healthy individuals. For this study, hemodialysis was performed before the doses of Remdesivir. Overall, there were no signs of drug-related toxicity in the patient, supporting Remdesivir's safety in patients with ESRD on hemodialysis (Sorgel et al. 2020).

Another observational study (Thakare, 2021) evaluated the safety of Remdesivir in 150 patients who had acute kidney injury (AKI) or chronic kidney disease (CKD) admitted to the ICU with COVID-19. 16\% of the participants had ESRD, and $46 \%$ of them received Remdesivir. The Patients received hemodialysis during the treatment, but the study did not specify if the sessions were pre or post Remdesivir doses. By the end of the study, no signs of toxicity were reported. The patients tolerated the drug appropriately, and $82 \%$ recovered while $18 \%$ died (Thakare, 2021).

In May of 2020, the US Food and Drug Administration (FDA) issued an Emergency Use Authorization for the use of Remdesivir in patients with COVID-19 (Office of the commission, 2020). Since Remdesivir is substantially insoluble in water (National Library of Medicine, 2021), its formulations contain SBECD as a solubility enhancer; this transporter molecule has been associated with liver and kidney toxicity. Consequently, the FDA recommendations suggest using Remdesivir in patients with (eGFR) $<30 \mathrm{~mL} / \mathrm{min} / 1.73 \mathrm{~m}^{2}$ only when the benefits outweigh the risk.

Several studies on SBECD as a vehicle for intravenous voricozonale show that in patients with (eGFR) $<30 \mathrm{~mL} / \mathrm{min} / 1.73 \mathrm{~m}^{2}$, the drug can be used, but hemodialysis is needed to maintain non-toxic concentrations of the drug (Dashti-Khavidaki, 2020).

In this literature review, case reports and observational studies utilize Remdesivir in severe presentations of COVID19 and with patients in the ICU where the benefits outweigh the risk. Clinicians maintained hemodialysis in the ESRD patients throughout the treatment with Remdesivir, measured the drugs and metabolite concentration, and performed analysis to evaluate renal parameters. In all cases, no toxicity was found, and patients improved. This leaves the question: is it safe to use Remdesivir on ESRD with intermittent hemodialysis? It is still too soon to conclude; however, a recent study evaluated the possibility. A case study (Le, 2020) reported intermittent hemodialysis's benefits in patients undergoing treatment 
with Remdesivir. A 40-year-old man with a history of double lung transplant a year prior was diagnosed with COVID-19. Rapid clinic deterioration necessitated transfer to the ICU. On day 21, intravenous Remdesivir infusion started, and 24 days after, progressive deterioration of renal function was observed. The team decided to initiate hemodialysis and measure the concentrations of GS441524 after 24 hours of the last doses and 4-hours after the hemodialysis session. They found a lower concentration of Remdesivir and GS-441524 after dialysis. This was the first study to show the impact of intermittent hemodialysis on Remdesivir and GS-441524 plasma exposures.

Though the question remains, the information available now seems favorable for the use of Remdesivir in patients with ESRD as long as the patient undergoes hemodialysis (Miller-Handley, 2020).

\section{CONCLUSIONS}

COVID-19 is a new disease, efforts have managed to determine some of the disease's pathophysiology, but much is still unclear. The approval of drugs for the treatment of COVID-19 represents a promising improvement towards decreasing mortality and complications of the disease. Remdesivir has shown in clinical trials reduction of hospitalization and recovery days; however, it is not recommended in ESRD patients. More studies, particularly randomized controlled trials, are needed to prove the drug's safety in this group of patients. However, available literature shows that it can be safe when combined with hemodialysis and constant Remdesivir concentration monitoring.

Study Limitation: Minimal Randomized clinical trials were available as at the time of this publication.

Conflict of Interest: There was no conflict of interest during this review.

\section{Reference}

[1] Food and Drug Administration. (2021, February 4) Frequently Asked Questions for Veklury (Remdesivir) Retrieved March 21, 2021, from

https://www.fda.gov/media/137574/download\#: :t ext=A.,of\%20COVID\%2D19\%20requiring\%20hospital ization

[2] Gelburd, R. (November 11 2020). Links between comorbidities, mortality detailed in FAIR Health Study. AJMC.

[3] Kooman, J., \& Van der Sande, F. (2020, December 15). COVID-19 in ESRD and Acute Kidney Injury. Retrieved From

https://www.ncbi.nlm.nih.gov/pmc/articles/PMC780 $2200 /$

[4] Scavone C, B. S. (April 2020). Current pharmacological treatment for Covid-19: What's next? British Journal of Pharmacology, 1476-5381.

[5] Adamsick, M. L. (2020, July 1). Remdesivir in Patients with Acute or Chronic Kidney Disease and COVID-19. American Society of Nephrology.

https://jasn.asnjournals.org/content/31/7/1384.long

[6] Adamsick, M. L. (2020, July 1). Remdesivir in Patients with Acute or Chronic Kidney Disease and COVID-19. American Society of Nephrology.

https://jasn.asnjournals.org/content/31/7/1384.long
[7] WHO. (2021). Weekly epidemiological update January 27, 2021. Retrieved from

https://www.who.int/publications/m/item/weeklyepidemiological-update---27-january-2021

[8] Food and Drug Administration. (2020, October). US Food and Drug Administration: Fact Sheet for Health Care Providers Emergency Use Authorization (EUA) of Remdesivir (GS-5734 ${ }^{\mathrm{TM}}$ ), Silver Spring, MD, US Food and Drug Administration, 2020. Retrieved from https://www.fda.gov/media/137566/download

[9] Beigel, J. H. (2020, November 5). Remdesivir for the Treatment of Covid-19 - Final Report. PubMed. https://pubmed.ncbi.nlm.nih.gov/32445440/

[10] Adamsick, M. L. (2020, July 1). Remdesivir in Patients with Acute or Chronic Kidney Disease and COVID-19. American Society of Nephrology.

https://jasn.asnjournals.org/content/31/7/1384.long

[11] Food and Drug Administration. (2020, October). US Food and Drug Administration: Fact Sheet for Health Care Providers Emergency Use Authorization (EUA) of Remdesivir (GS-5734 $4^{\mathrm{TM}}$ ), Silver Spring, MD, US Food and Drug Administration, 2020. Retrieved from https://www.fda.gov/media/137566/download

[12] Kiser, T., Fish, D., Aquilante, C., Rower, J., Wempe, F., Maclaren, R., \& Teitelbaum, I. (2015, February 3). Evaluation of sulfobutylether- $\beta$-cyclodextrin (SBECD) accumulation and voriconazole pharmacokinetics in critically ill patients undergoing continuous renal replacement therapy. Retrieved from

https://www.ncbi.nlm.nih.gov/pmc/articles/PMC43386 18/

[13] Hoover, R., Alcorn, H., Lawrence, L., Paulson, S., Quintas, M., Luke, D., \& Cammarata, S. (2018, June). Clinical Pharmacokinetics of Sulfobutylether- $\beta$-Cyclodextrin in Patients with Varying Degrees of Renal Impairment. Retrieved from

https://www.ncbi.nlm.nih.gov/pmc/articles/PMC671 8009/

[14] Hafner, V. (2010, June 1). Pharmacokinetics of Sulfobutylether-Beta-Cyclodextrin and Voriconazole in Patients with End-Stage Renal Failure during Treatment with Two Hemodialysis Systems and Hemodiafiltration. Antimicrobial Agents and Chemotherapy. https://aac.asm.org/content/54/6/2596?ijkey=3011 683666ce03c14c5e8822e578becf3c408020\&keytype 2=tf_ipsecsha

[15] Gérard, A. O., Lurain, A., Fresse, A., Parassol, N., Muzzone, M., Rocher, F., Esnault, V. (2020). Remdesivir and Acute Renal Failure: A Potential Safety Signal From Disproportionality Analysis of the WHO Safety Database. PubMed. https://pubmed.ncbi.nlm.nih.gov/33340409/

[16] Aiswarya, D. (2020a). Use of Remdesivir in Patients With COVID-19 on Hemodialysis: A Study of Safety and Tolerance.PubMed. https://pubmed.ncbi.nlm.nih.gov/33354635/

[17] Sörgel, F. (2021, February 11). Pharmacokinetics of Remdesivir in a COVID-19 patient with end-stage renal disease on intermittent hemodialysis. PubMed. https://pubmed.ncbi.nlm.nih.gov/33251541/ 
[18] Thakare, S. (2020). Safety of Remdesivir in Patients with Acute Kidney Injury or CKD. PubMed. https://pubmed.ncbi.nlm.nih.gov/33073066/

[19] Use of Remdesivir in Patients With COVID-19 on Hemodialysis: A Study of Safety and Tolerance. (2021, March 1). ScienceDirect.

https://www.sciencedirect.com/science/article/pii /S2468024920318003

[20] Office of the Commissioner. (2020, August 28). COVID19 Update: FDA Broadens Emergency Use Authorization for Veklury (remdesivir) to Include All Hospitalized Patients for Treatment of COVID-19. US Food and Drug Administration.

https://www.fda.gov/news-events/pressannouncements/covid-19-update-fda-broadensemergency-use-authorization-veklury-remdesivirinclude-all-hospitalized
[21] National Library of Medicine. (2021, March 20). COMPOUND SUMMARY Remdesivir. Retrieved from

https://pubchem.ncbi.nlm.nih.gov/compound/ Remdesivir

[22] Dashti-Khavidaki, S., Khalili, H., Nourian, A. (2020). Pharmacotherapy Considerations in CKD Patients With COVID-19, A Narrative Review. PubMed.

https://pubmed.ncbi.nlm.nih.gov/32655019/

[23] Lê, M. P., Le Hingrat, Q., Jaquet, P., Wicky, P., Bunel, V., Massias, L., Visseaux, B. (2020, October 20). Removal of Remdesivir's Metabolite GS441524 by Hemodialysis in a Double Lung Transplant Recipient with COVID-19. PubMed. https://pubmed.ncbi.nlm.nih.gov/32868327/

[24] Miller-Handley, H., Luckett, K., Govil, A. (2020). Treatment Options for Coronavirus Disease 2019 in Patients with Reduced or Absent Kidney Function. PubMed.

https://pubmed.ncbi.nlm.nih.gov/33308510/ 